\title{
Potential virulence of Klebsiella sp. isolates from enteral diets
}

\author{
S.C.L. Pereira ${ }^{1}$ and M.C.D. Vanetti ${ }^{2}$ \\ ${ }^{1}$ Departamento de Nutrição, Escola de Enfermagem, Universidade Federal de Minas Gerais, \\ Belo Horizonte, MG, Brasil \\ ${ }^{2}$ Departamento de Microbiologia, Universidade Federal de Viçosa, Viçosa, MG, Brasil
}

\begin{abstract}
We aimed to evaluate the potential virulence of Klebsiella isolates from enteral diets in hospitals, to support nosocomial infection control measures, especially among critical-care patients. Phenotypic determination of virulence factors, such as capsular expression on the external membrane, production of aerobactin siderophore, synthesis of capsular polysaccharide, hemolytic and phospholipase activity, and resistance to antibiotics, which are used therapeutically, were investigated in strains of Klebsiella pneumoniae and K. oxytoca. Modular industrialized enteral diets (30 samples) as used in two public hospitals were analyzed, and Klebsiella isolates were obtained from six (20\%) of them. The hypermucoviscous phenotype was observed in one of the K. pneumoniae isolates (6.7\%). Capsular serotypes K1 to K6 were present, namely K5 and K4. Under the conditions of this study, no aerobactin production, hemolytic activity or lecithinase activity was observed in the isolates. All isolates were resistant to amoxicillin and ampicillin and sensitive to cefetamet, imipenem, chloramphenicol, gentamicin and sulfamethoxazole-trimethoprim. Most $K$. pneumoniae isolates $(6 / 7,85.7 \%)$ from hospital B presented with a higher frequency of resistance to the antibiotics tested in this study, and multiple resistance to at least four antibiotics (3/8; $37.5 \%)$ compared with isolates from Hospital A. The variations observed in the antibiotic resistance profiles allowed us to classify the Klebsiella isolates as eight antibiotypes. No production of broad-spectrum $\beta$-lactamases was observed among the isolates. Our data favor the hypothesis that Klebsiella isolates from enteral diets are potential pathogens for nosocomial infections.
\end{abstract}

Key words: Klebsiella; Enteral diets; Pathogenicity

\section{Introduction}

Bacteria of the genus Klebsiella frequently cause nosocomial infections and are associated with high morbidity and mortality in people (1). K. pneumoniae, in particular, is the most clinically important Klebsiella species and occurs in nosocomial infections, such as those of the urinary tract, and in cases of pneumonia and septicemia (1).

Given the severity of the worldwide situation regarding nosocomial infections, control efforts should be directed at identifying the sources of contamination and means of transmission to implement preventive measures (2). The gastrointestinal tract and the hands of hospital medical staff are recognized as sources of contamination by Klebsiella $(3,4)$. These bacteria are also found frequently in enteral diets. However, the involvement of such foods as sources of this opportunistic pathogen has received little attention in the scientific and medical literature $(5,6)$.
Recently, a study identified $K$. pneumoniae as the nosocomial pathogen responsible for a large-scale outbreak, which occurred via the hospital's own food supply chain (7). This was the first study to address the transmission of multidrug-resistant Klebsiella through food in the hospital environment. Furthermore, according to Podschun et al. (8) K. pneumoniae isolates from nonclinical sources are, in common with clinical isolates, able to express virulence factors. Among the factors that contribute to the pathogenicity of Klebsiella, the nature of the polysaccharide capsule of the external membrane, production of siderophores and fimbrial adhesins, and synthesis of capsular polysaccharide (as characterized by the mucoid appearance of the colonies) are considered to underlie this virulence process (8-11).

Other factors, such as hemolysin and phospholipase production, as well as multidrug resistance

Correspondence: S.C.L. Pereira: <simoneclpereira@gmail.com>.

Received August 13, 2014. Accepted April 15, 2015. First published online July 10, 2015. 
against the antibiotics used for therapy, are considered to be intensifiers of the virulence potential of Klebsiella (9). Moreover, the number of outbreaks of nosocomial infections caused by multidrug-resistant Klebsiella isolates, especially extended-spectrum beta-lactamase (ESBL) producers, have increased over the last few years $(12,13)$.

Despite this, no studies on enteral diets similar to the study described herein were found in the scientific literature we consulted. Therefore, the objective of the present study was to investigate the pathogenicity of the Klebsiella strains isolated from modular enteral diets, by analyzing their phenotypic expression of virulence factors and their antibiotic resistance profiles.

\section{Material and Methods}

\section{Origin of the isolates}

Modular industrialized (milk-based and food supplement) enteral diets (30 samples) as used in two public hospitals in the State of Minas Gerais (Brazil) were analyzed, and Klebsiella sp. isolates were obtained from six $(20 \%)$ of them. The isolates were analyzed for their potential virulence and the results are reported in Table 1. We emphasize that the samples were collected before administration to the patients.

\section{Phenotypic determination of virulence factors}

Mucoid appearance, presence of capsules, and serotyping. The mucoid appearance of the colonies was observed after incubation $\left(37^{\circ} \mathrm{C}, 48 \mathrm{~h}\right)$ of the Klebsiella isolates in brain heart infusion $(\mathrm{BHI})$ agar (Difco Laboratories, USA). Individual colonies were tested for their capacity to form viscous chains. Chain formation (length $>5 \mathrm{~mm}$ ) was an indicator of a positive phenotype for hypermucoviscosity (14).

The presence of capsules in the Klebsiella isolates was observed on slides treated with alcoholic Congo red $(0.2 \%$ solution; SRL Chemicals, India) using an optical microscope (oil immersion lens; Leica DMLS, Leica Microsystems, Germany). The unstained region seen on a blue background around the red central nucleus of the bacterial cells indicated the presence of a capsule (10). Additionally, the K. pneumoniae isolates were sent to the Laboratório de Enterobactérias, Departamento de Bacteriologia, Fundação Oswaldo Cruz, Rio de Janeiro, RJ, Brazil for immunological analysis of the serotypes with regard to the K1 to K6 capsular antigens of the external membrane, as determined by antigen-antibody reaction analysis.

Analysis of aerobactin production. Aerobactin production was demonstrated by using the LG 1522 strain of Escherichia coli as an indicator. This mutant is deficient in aerobactin synthesis, but has the aerobactin receptor (11).

Table 1. Virulence phenotypes of Klebsiella isolates from modular enteral diets.

\begin{tabular}{|c|c|c|c|}
\hline \multirow[t]{2}{*}{ Isolate/origin } & \multirow[t]{2}{*}{ Specie } & \multicolumn{2}{|c|}{ Virulence phenotypes } \\
\hline & & Mucoid aspect & Capsular serotype \\
\hline $\mathrm{P} 1 / \mathrm{A}$ & K. oxytoca & Absent & SND \\
\hline $\mathrm{P} 2 / \mathrm{A}$ & K. oxytoca & Absent & SND \\
\hline $\mathrm{P} 3 / \mathrm{A}$ & K. oxytoca & Absent & SND \\
\hline $\mathrm{P} 4 / \mathrm{A}$ & K. oxytoca & Absent & SND \\
\hline P5/A & K. oxytoca & Absent & SND \\
\hline P6/A & K. pneumoniae & Weak & $\mathrm{SNH}$ \\
\hline P7/A & K. pneumoniae & Weak & $\mathrm{SNH}$ \\
\hline $\mathrm{P} 8 / \mathrm{A}$ & K. pneumoniae & Weak & $\mathrm{SNH}$ \\
\hline $\mathrm{P} 9 / \mathrm{A}$ & K. oxytoca & Absent & SND \\
\hline $\mathrm{P} 11 / \mathrm{A}$ & K. pneumoniae & Weak & $\mathrm{SNH}$ \\
\hline $\mathrm{P} 13 / \mathrm{A}$ & K. pneumoniae & Moderate & $\mathrm{K} 5$ \\
\hline $\mathrm{P} 14 / \mathrm{A}$ & K. pneumoniae & Weak & $\mathrm{SNH}$ \\
\hline $\mathrm{P} 15 / \mathrm{A}$ & K. pneumoniae & Weak & $\mathrm{SNH}$ \\
\hline P17/A & K. pneumoniae & Weak & $\mathrm{SNH}$ \\
\hline U1/B & K. pneumoniae & Moderate & $\mathrm{K} 5$ \\
\hline U2/B & K. pneumoniae & Weak & $\mathrm{SNH}$ \\
\hline U3/B & K. pneumoniae & Moderate & $\mathrm{SNH}$ \\
\hline U4/B & K. pneumoniae & Intense & K5 \\
\hline U5/B & K. pneumoniae & Weak & K4 \\
\hline U7/B & K. pneumoniae & Absent & $\mathrm{SNH}$ \\
\hline U8/B & K. pneumoniae & Moderate & $\mathrm{K} 5$ \\
\hline
\end{tabular}

A: Hospital A; B: Hospital B; SND: serotype not determined. SNH: serotyping nonspecific but higher than K6. See Material and Methods for details. 
This bacterium was cultivated in Petri dishes containing M9 agar, with addition of $200 \mu \mathrm{M} 2,2^{\prime}$-dipyridine (Sigma Chemical Co., USA). One colony of each Klebsiella isolate to be tested was inoculated into the agar medium containing the indicator strain. Aerobactin-producing strains were viewed as a halo of LG 1522 E. coli cell growth around the inoculated isolates. The $E$. coli strain LG 1315, which is genotypically (ara entA lac leu $\mathrm{mtL}$ proC $r p s L$ supE thi tonA trpE $x y l$ ) and phenotypically certified as an aerobactin producer, was the positive control. The $E$. coli strain HB101, which is certified as an aerobactin nonproducer strain, was the negative control (15).

Phosphatidylcholine-specific phospholipase C (PC-PLC). PC-PLC activity was determined using the method described by Coffey et al. (16). Klebsiella cultures were grown in $\mathrm{BHI}$ broth (4 mL; Difco Laboratories) and incubated at $37^{\circ} \mathrm{C}$ for 18-24 h. Aliquots $(3 \mu \mathrm{L})$ from each culture were inoculated onto the surface of $\mathrm{BHI}$ agar containing $2 \% \mathrm{NaCl}$ and $5 \%$ egg yolk emulsion, previously diluted in $0.85 \% \mathrm{NaCl}$ $(1: 1, \mathrm{v} / \mathrm{v})$. The plates, incubated $\left(37^{\circ} \mathrm{C}, 96 \mathrm{~h}\right)$ in a Gas-Pak jar with an anaerobiosis generator (AnaeroGen ${ }^{\mathrm{TM}}$, Oxoid, UK), were observed to determine whether a lecithin hydrolysis halo around the colonies was present.

Hemolysin activity. The hemolytic activity of the Klebsiella cultures was investigated using blood agar, as described by Kokosharov and Phetisova (17). BHI plates (Oxoid) containing 5\% defibrinated rabbit blood were prepared and small wells $(3 \mathrm{~mm})$ were produced. The wells were used to inoculate $5 \mu \mathrm{L}$ bacterial suspensions, after which the plates were incubated at $37^{\circ} \mathrm{C}$. The plates were observed after anaerobic incubation at $37^{\circ} \mathrm{C}$ for $24 \mathrm{~h}$ and the presence of a partial halo or total hemolysis was considered a hemolysin-positive result.

\section{Antibiotic susceptibility}

The susceptibility of each Klebsiella isolate to antimicrobial agents was determined through the disk diffusion method in Mueller-Hinton agar (Difco Laboratories), according to the guidelines of the Clinical and Laboratory Standards Institute (CLSI) (18). The antibiotics (Sensibiodisc-CECON, Brazil) used in this study were as follows: nalidixic acid (NAL, $30 \mu \mathrm{g}$ ), amikacin (AMI, $30 \mu \mathrm{g}$ ), amoxicillin (AMO, $10 \mu \mathrm{g})$, amoxicillin-clavulanic acid (AMC, $30 \mu \mathrm{g} / 10 \mu \mathrm{g}$ ), ampicillin (AMP, $10 \mu \mathrm{g}$ ), chloramphenicol (CLO, $30 \mu \mathrm{g}$ ), gentamicin (GEN, $10 \mu \mathrm{g}$ ), imipenem (IMP, $30 \mu \mathrm{g})$, kanamycin (KN, $30 \mu \mathrm{g}$ ), neomycin (NO, $30 \mu \mathrm{g})$, tetracycline (TET, $30 \mu \mathrm{g})$, ticarcillin-clavulanic acid (TIC, $75 \mu \mathrm{g} / 10 \mu \mathrm{g}$ ), and trimethoprim-sulfamethoxazole (SUT, 1.25/23.75 $\mu \mathrm{g}$ ). To identify ESBL-producing strains, the cultures were screened with the following antibiotics: cefotaxime (CTX, $30 \mu \mathrm{g})$, ceftazidime (CAZ, $30 \mu \mathrm{g}$ ), cefetamet (CEF, $10 \mu \mathrm{g})$, cefalotin, (CFL; $30 \mu \mathrm{g})$, and aztreonam (ATM, $30 \mu \mathrm{g}$ ), according to the CLSI guidelines (18). After incubation $\left(35^{\circ} \mathrm{C}, 16-18 \mathrm{~h}\right)$ with cefetamet, ceftazidime, aztreonam, cefotaxime, and cefalotin, the isolates with growth-inhibition halos were suspected to be
ESBL-producing strains. The $\mathrm{E}$ test (AB BIODISK, Sweden) was used as a confirmatory test, and ceftazidime and cefotaxime antibiotics were used as substrates. Reduction in the minimum inhibitory concentration (MIC) of the antimicrobial agent in association with the betalactamase inhibitor was compared with the MIC of the antimicrobial agent beta-lactamase alone (ceftazidime or cefotaxime) and was indicative of ESBL-producing strains.

\section{Results}

We analyzed 30 samples from the diets used in public hospitals and identified 21 Klebsiella isolates. Most $(n=15$; $71.4 \%$ ) of them were of the $K$. pneumonia species. The $K$. oxytoca species was identified in one of the hospitals (Table 1).

Most $(42.9 \%)$ of the Klebsiella isolates obtained from the modular enteral diets formed colonies with a low mucoid appearance in $\mathrm{BHI}$ agar. Four isolates $(26.7 \%)$ of K. pneumoniae had a moderate mucoid appearance and only the isolate U4 $(6.7 \%)$ presented a hypermucoviscosity phenotype. The presence of a capsule in the isolate U4 and isolates with colonies of moderate mucoid appearance were confirmed by observing the preparations under a microscope. In isolates whose colonies presented a low mucoid appearance, no capsule could be observed through the staining used in this study. Additionally, five isolates with the presence of capsule antigens between $\mathrm{K} 1$ and $\mathrm{K} 6$ were identified (Table 1).

Under our experimental conditions, the Klebsiella isolates from the enteral diets we tested did not exhibit aerobactin production (Figure 1), hemolytic activity, or lecithinase activity.

All the Klebsiella isolates (21) from the enteral diets were resistant to amoxicillin and ampicillin (Table 2). Six isolates $(28.6 \%)$ were shown to be resistant when amoxicillin was used in association with the beta-lactamase inhibitor, clavulanic acid (Table 2). This behavior was also observed in relation to the association between ticarcillin and clavulanic acid, albeit less frequently (Table 2).

The Klebsiella isolates were sensitive to beta-lactamic group antibiotics, namely cefetamet, which is a thirdgeneration cephalosporin, and imipenem, which is a carbapenem antibiotic (Table 2). These isolates were also inhibited by different groups of antibiotics, such as chloramphenicol (a quinolone), gentamicin (an aminoglycoside), and sulfatrim (a mixture of trimethoprim and sulfamethoxazole).

The $K$. pneumoniae isolates found in the enteral diets from hospital B showed a high frequency of resistance to the antibiotics tested and multiple resistance to at least four antibiotics compared with the isolates from hospital $A$ (Table 2).

The differences observed in the antibiotic resistance profiles among the Klebsiella isolates allowed us to classify them into the antibiotypes described in Table 3. 


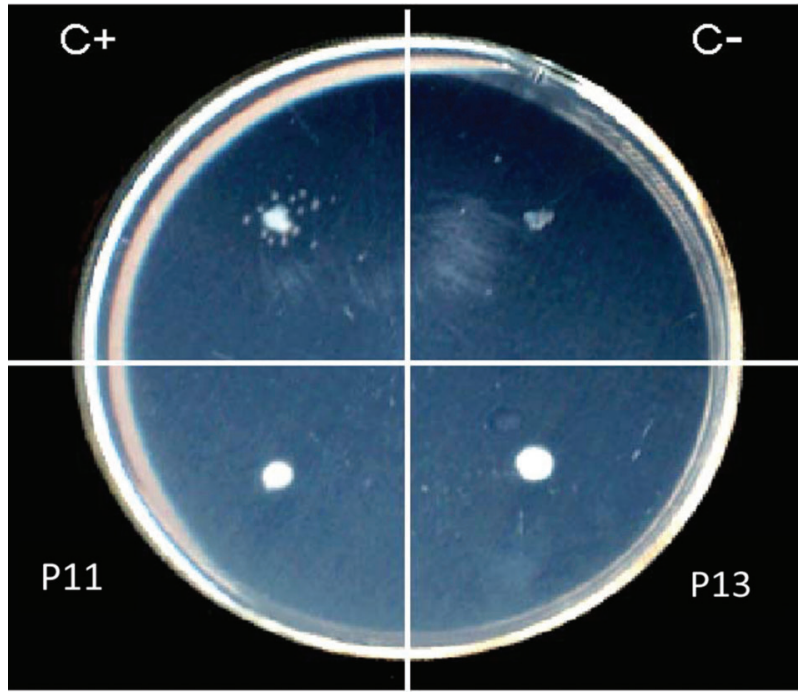

Figure 1. Aerobactin production on M9 agar with $200 \mu \mathrm{g}$ 2,2'bipyridine. A positive test is indicated by the presence of satellite colonies around the isolate, as seen in the positive control $(\mathrm{C}+)$. $\mathrm{C}-$ : negative control. P11 and P13 isolates are described in Table 1.

ESBL production by the $K$. pneumoniae and $K$. oxytoca isolates was not observed. This observation could be made because no reduction was seen in the MIC of the antimicrobial agent in association with the beta-lactamase inhibitor in comparison with that of the beta-lactamic antimicrobial agent alone (ceftazidime or cefotaxime). Resistance to these antibiotics, which were used to detect ESBL-producing strains, was in the range $0-38.1 \%$ (Table 2). The highest frequencies of resistance were observed for the beta-lactamic agents, aztreonam $(38.1 \%)$ and cefotaxime $(33.3 \%)$, whereas the lowest frequencies of resistance were recorded for ceftazidime $(4.8 \%)$ and cefetamet $(0 \%)$ (Table 2$)$.

\section{Discussion}

In this study, we found the frequency of the hypermucoviscous phenotype among the colonies formed by $K$. pneumonia isolates to be low $(6.7 \%)$. However, this finding deserves further scrutiny. This is because some studies conducted on clinical samples have reported a low frequency for this phenotype. For example, Vernet et al. (19) observed that only $7.0 \%$ of $241 \mathrm{~K}$. pneumoniae clinical isolates had this phenotype. In a study with greater sample coverage (20), $455 \mathrm{~K}$. pneumoniae clinical isolates were found to be involved in consecutive cases of bacteremia in seven countries. In two of these countries, a very high frequency of the hypermucoviscous phenotype was found in isolates that caused pneumonia (94\%) and invasive syndrome (100\%). However, in other countries the mean frequency was $2 \%$ (i.e., a lower frequency than that found in the present study).

The mucoid appearance of Klebsiella colonies is related to the production of a capsule, a virulence factor essential for Klebsiella to overcome a host's immune

Table 2. Resistance of Klebsiella isolates to various antimicrobial agents used in therapy.

\begin{tabular}{|c|c|c|c|c|}
\hline \multirow[t]{2}{*}{ Antibiotics } & \multicolumn{4}{|c|}{ Species/Hospital } \\
\hline & K. pneumoniae/A & K. oxytoca/A & K. pneumoniae/B & Total \\
\hline Amoxicillin + clavulanic acid (AMC) & $2(25.0)$ & $0(0.0)$ & $4(57.1)$ & $6(28.6)$ \\
\hline Nalidixic acid (NAL) & $2(25.0)$ & $0(0.0)$ & $6(85.7)$ & $8(38.1)$ \\
\hline Amikacin (AMI) & $1(12.5)$ & $0(0.0)$ & $0(0.0)$ & $1(4.8)$ \\
\hline Amoxicillin (AMO) & $8(100.0)$ & $6(100.0)$ & $7(100.0)$ & $21(100.0)$ \\
\hline Ampicillin (AMP) & $8(100.0)$ & $6(100.0)$ & $7(100.0)$ & $21(100.0)$ \\
\hline Aztreonam (ATM ) & $2(25.0)$ & $0(0.0)$ & $6(85.7)$ & $8(38.1)$ \\
\hline Cefalotin (CFL) & $2(25.0)$ & $0(0.0)$ & $5(71.4)$ & 7 (33.3) \\
\hline Cefetamet (CEF) & $0(0.0)$ & $0(0.0)$ & $0(0.0)$ & $0(0.0)$ \\
\hline Cefotaxime (CTX) & $2(25.0)$ & $0(0.0)$ & $5(71.4)$ & $7(33.3)$ \\
\hline Ceftazidime (CAZ) & $0(0.0)$ & $0(0.0)$ & $1(14.3)$ & $1(4.8)$ \\
\hline Chloramphenicol (CLO) & $0(0.0)$ & $0(0.0)$ & $0(0.0)$ & $0(0.0)$ \\
\hline Gentamicin (GEN) & $0(0.0)$ & $0(0.0)$ & $0(0.0)$ & $0(0.0)$ \\
\hline Imipenem (IPM) & $0(0.0)$ & $0(0.0)$ & $0(0.0)$ & $0(0.0)$ \\
\hline Kanamycin (KN) & $3(37.5)$ & $0(0.0)$ & $0(0.0)$ & $3(14.3)$ \\
\hline Neomycin (NO) & $2(25.0)$ & $0(0.0)$ & $1(14.3)$ & $3(14.3)$ \\
\hline Trimethoprim-sulfamethoxazole (SUT) & $0(0.0)$ & $0(0.0)$ & $0(0.0)$ & $0(0.0)$ \\
\hline Tetracycline (TET) & $2(25.0)$ & $0(0.0)$ & $3(42.9)$ & $5(23.8)$ \\
\hline Ticarcillin + clavulanic acid (TIC) & $1(12.5)$ & $0(0.0)$ & $0(0.0)$ & $1(4.8)$ \\
\hline
\end{tabular}

Data are reported as number with percentage in parentheses. A: Hospital A; B: Hospital B. See Material and Methods for details. 
Table 3. Antibiotypes of Klebsiella isolates from modular enteral diets.

\begin{tabular}{lcc}
\hline Antibiotype & Isolates $^{*}$ & Antibiotic resistance \\
\hline I & P1, P2, P3, P4, P5, P6, P7, P8, P9, P11, & AMO and AMP \\
II & P13, U5 & NAL, AMO, AMP, ATM \\
III & U3 & AMO, AMP, ATM, KN, TET \\
IV & P15 & AMC, NAL, AMO, AMP, ATM, CFL, CTX \\
V & U2 & NAL, AMO, AMP, ATM, CFL, CTX, TET \\
VI & U4 & AMC, NAL, AMO, AMP, CFL, CTX, KN, NO \\
VII & P17 & AMC, NAL, AMO, AMP, ATM, CFL, CTX, CAZ \\
VIII & U1 & AMC, NAL, AMO, AMP, ATM, CFL, CTX, TET \\
IX & U7 & AMC, NAL, AMO, AMP, ATM, CFL, CTX, NO, TET \\
X & U8 & P14 \\
\hline
\end{tabular}

* The isolates are specified in Table 1 and the antibiotics are described in Table 2. See Material and Methods for details.

system (21). Furthermore, a recent study attributed the role of the magA and $r m p A$ genes as determinants of the hypermucoviscous phenotype in the pathogenesis of the K1 capsular serotype of $K$. pneumoniae, which is involved in bacteremia (22). The $r m p A$ gene, which codes for a positive regulator of extracapsular mucopolysaccharide expression (20), was also identified in two (2/54) clinical isolates out of a total of five isolates with the hypermucoviscous phenotype. However, the isolates were not serotyped for K1-K6 positivity (9). The hypermucoviscous phenotype is seen predominantly in strains with the $\mathrm{K} 1$ and $\mathrm{K} 2$ serotypes; these are the clinical isolates causing invasive diseases. This would explain the low hypermucoviscosity rates found among the isolates in the present study (9).

The presence of a capsule is an important virulence factor relating to the severity of an infection. A capsule is generally present in clinical strains, and 78 capsule types were identified in K. pneumoniae. However, because of the limitations of traditional serotyping methods, capsule type determination is often difficult (23). In the present study, isolates were found to have the K5 (4) and K4 (1) serotypes out of a possible range of six main serotypes (K1-K6) for this microbial species. Among the 78 distinct capsule antigens of $K$. pneumoniae, only the $\mathrm{K} 1, \mathrm{~K} 2$, and K5 antigens are considered highly virulent, being frequently associated with severe infections in humans and animals. One of these types of antigen was found in four of the isolates in this study.

None of the isolates we tested exhibited aerobactin production. According to some authors $(8,9,19)$, Klebsiella isolates with aerobactin-positive phenotypes are rare, irrespective of the species or isolation source.

Aerobactin is one of the siderophores (extracellular ferric chelating agents) secreted by bacterial cells; hence, it can eliminate free $\mathrm{Fe}^{3+}$ ions from iron-binding proteins in the host (9). These siderophores play a critical role in bacterial virulence. Based on our results and the information found in the scientific literature (19), Klebsiella should be included in the group of enterobacteria that present low aerobactin production rates $(<20 \%)$. In this regard, and given that iron bioavailability in the host is extremely low $\left(10^{-18} \mathrm{M}\right)$, iron is essential for microbial growth, and the incidence of the aerobactin phenotype among Klebsiella isolates is low, we raise the question of which mechanisms in the host would ensure iron supply to these bacteria $(8,9,19)$.

It has been shown that enterobactin, another ferric chelating agent, is present in most clinical isolates of $K$. pneumoniae $(9,15)$. The role of enterobactin in Klebsiella virulence is still uncertain, but expression of the enterobactin gene seems to be activated during infection. A recent study found the presence of genes that code for synthesis of enterobactin in clinical isolates of noninvasive infections (bacteremia) (9).

The absence of hemolytic activity found under the conditions of this study, which are often not reproducible under in vitro conditions, may be related to a lack of activators for expression of genes that code for this enzyme (24). The hemolysin virulence factor was first detected by Albesa (1989) in a test in which blood agar containing rabbit erythrocytes was used (24). Since then, this investigator and her group have been studying this cytolysin, and its specificity for rabbit erythrocytes has been confirmed. Hemolysin is a pore-forming toxin that makes some nutrients, such as the iron ion in hemoglobin, available. In a recent study, none of the 54 clinical isolates presented with hemolysis or the presence of the hlyA and cnf-1 genes encoding this activity. This is consistent with the lack of studies reporting the genotypic and phenotypic presence of hemolysis in Klebsiella, although hemolytic activity has been described $(24,25)$. Lecithinase or PC-PLC activity was absent in all isolates under the conditions in which this was determined. This result is in agreement with that of Singh et al. (26) who reported that lecithinase activity in Klebsiella isolates was rare (only 
$2.4 \%$ of their 168 clinical isolates of $K$. pneumoniae presented such activity). Also, they found that lecithinase activity was absent among the 11 isolates of $K$. oxytoca. Their study was the first report on lecithinase activity in Klebsiella isolates, and the role of this virulence factor in the pathogenicity of this genus of bacteria has not yet been fully clarified.

Regarding the antibiotics used in the present study, a high prevalence of resistance to ampicillin and amoxicillin was observed. Resistance to ampicillin was also observed in a study on clinical isolates of $K$. pneumonia (27) and in a study on isolates of this enterobacterium in expressed human breast milk (28).

Additionally, the prevalence of resistance to multiple antibiotics among isolates from hospital B (85.7\%) was higher than among isolates from hospital A (37.5\%). According to Davies (27), the prevalence of antibiotic resistance between different hospitals may vary because of local epidemiological factors such as control of the use of antibiotics and the preventive measures applied by hospital infection committees in relation to multidrug resistant strains. Therefore, strategies to prevent nosocomial emergence and spread of antibiotic-resistant organisms are essential. In this sense, improvement in hospital infection control practices outlined for prevention and transmission of multidrug resistant microorganisms is necessary, especially in hospital B. A combination of epidemiological surveillance, molecular genotyping, observational studies on compliance, and mathematical modeling will improve our ability to determine the quantitative effects of individual infection control measures. This may help us to design more effective infection control programs (29-32).

Here, no correlation between the high incidence of resistance to cefotaxime and ESBL production was observed. This is an important clinical result, although the isolates were multiply resistant to up to 12 antibiotics.

The condition of multidrug resistance observed in the present study justifies the assertion that bacterial resistance to antimicrobial agents is a serious public health problem. This is the real situation within which control initiatives have been proposed. In Brazil, legally established control over medications based on antimicrobial agents for use under medical prescription, either singly or in combination, was initiated in 2010. Thus, these substances can now be dispensed only through a specially controlled prescription (33).

Moreover, the observation that Klebsiella isolates are resistant to multiple antibiotics has significant clinical importance. This is because enteral diets are prescribed for patients who are highly susceptible to nosocomial infections and are concentrated in intensive care units. Such patients are subject to the intrinsic risks related to complex underlying diseases, nutritional vulnerability, extreme ages (premature infants and elderly people), and the effects of drug treatments, in addition to extended-spectrum antimicrobial agents. Furthermore, given that hospitals have complex organizational structures, patients are subject to extrinsic risks such as prolonged stays in hospital environments and routine invasive procedures, the quality of which is not guaranteed for all hospital services (12). In this context, the risk to health becomes more severe because this environment is a potential source of genes for resistance to pathogenic microorganisms, especially those that cause highly toxic infections. Additionally, the intestinal tract is an appropriate niche for transference of genes related to resistance (34).

Some authors have suggested that transfer of plasmids that carry more than one antimicrobial resistance gene facilitates horizontal gene transmission of different resistance mechanisms to other bacteria, thus further worsening the existing problem of multiple resistance to antibiotics in hospital environments $(27,35)$. Such transfers are probably more effective when hosts are simultaneously subjected to selective pressure from an antimicrobial substance to which the microorganisms involved are resistant. From this perspective, hospitals are environments where drugresistant microorganisms and others that determine such resistance are more likely to be present. Thus, in such environments, horizontal gene transfer has a greater chance of occurring and the consequences may be more severe in terms of therapeutic failure $(36,37)$.

The occurrence of chromosomal mutations alone does not explain the rapid acquisition of genetic resistance to antibiotics in bacteria. It is established that plasmids are particularly important in the evolution of antibiotic-resistant bacteria. It has also been suggested that transfer of plasmids carrying resistance genes facilitates horizontal gene transmission to other bacteria $(23,27)$. Food seems to be an effective source for human acquisition of resistant bacteria and genes for resistance to drugs, but the extent and real consequences of this type of exposure have not yet been fully investigated (38).

The data obtained in this study favor the hypothesis that Klebsiella isolates from enteral diets are potential pathogens for nosocomial infections, especially in criticalcare patients. This is because capsular serotypes, which are only identified in severe cases of nosocomial infection, were observed among the isolates in our study. They include the $\mathrm{K} 5$ and $\mathrm{K} 4$ serotypes, which are among the serotypes of clinical importance (K1 to K6). Additionally, the presence of the hypermucoviscous phenotype was observed, thus increasing the probability of an invasion process occurring in cases of isolates with one of these important capsular serotypes. This profile of virulence, which may provide a partial explanation for infection by Klebsiella, is a risk for microbiological security. This situation occurs during propagation of infection in intensive care units, where the intrinsic and extrinsic risks favor nosocomial infections. The synergy that may exist between Klebsiella virulence factors should be highlighted, especially when it occurs in combination with resistance to the 
multiple types of antibiotic used for therapeutic use, as has also been shown in the present study. Further studies are necessary to assess the pathogenicity of Klebsiella isolates under in vivo conditions.

The present results also showed that control measures against nosocomial infections are important for those handling enteral diets. Regarding individual and collective practices, participation of all sectors of the hospital in the control of this situation is necessary. We emphasize that rigorous and systematic monitoring of the risks related to the origin of the food has to be applied to the hospital environment. Good food-handling practices are necessary to control the dissemination of Klebsiella and other bacteria in the hospital environment.

In this regard, our study adds new knowledge that supports preventative strategies against hospital acquired infections caused by Klebsiella and emphasizes the

\section{References}

1. Lin YT, Wang FD, Chan YJ, Fu YC, Fung CP. Clinical and microbiological characteristics of tigecycline non-susceptible Klebsiella pneumoniae bacteremia in Taiwan. BMC Infect Dis 2014; 14: 1, doi: 10.1186/1471-2334-14-1.

2. WHO. World Health Organization. Core components for infection prevention and control programs. Assessment tools for IPC programmes. Prevention of hospital-acquired infections. Geneve: World Health Organization; 2011.

3. Ekrami AR, Kayedani A, Jahangir M, Kalantar E, Jalali M. Isolation of common aerobic bacterial pathogens from the environment of seven hospitals, Ahvaz, Iran. Jundishapur J Microbiol 2011; 4: 75-82.

4. Westerbeek EA, van den Berg A, Lafeber HN, Fetter WP, van Elburg RM. The effect of enteral supplementation of a prebiotic mixture of non-human milk galacto-, fructo- and acidic oligosaccharides on intestinal permeability in preterm infants. Br J Nutr 2011; 105: 268-274, doi: 10.1017/S0007 14510003405.

5. Hurrell E, Kucerova E, Loughlin M, Caubilla-Barron J, Hilton A, Armstrong R, et al. Neonatal enteral feeding tubes as loci for colonisation by members of the Enterobacteriaceae. BMC Infect Dis 2009; 9: 146, doi: 10.1186/1471-2334-9-146.

6. Kanevsky-Mullarky I, Nedrow AJ, Garst S, Wark W, Dickenson M, Petersson-Wolfe CS, et al. Short communication: comparison of virulence factors in Klebsiella pneumoniae strains associated with multiple or single cases of mastitis. J Dairy Sci 2014; 97: 2213-2218, doi: 10.3168/ jds.2013-7140.

7. Calbo E, Freixas N, Xercavins M, Riera M, Nicolas C, Monistrol O, et al. Foodborne nosocomial outbreak of SHV1 and CTX-M-15-producing Klebsiella pneumoniae: epidemiology and control. Clin Infect Dis 2011; 52: 743-749, doi: $10.1093 / \mathrm{cid} / \mathrm{ciq} 238$.

8. Podschun R, Pietsch S, Holler C, Ullmann U. Incidence of Klebsiella species in surface waters and their expression of virulence factors. Appl Environ Microbiol 2001; 67: 33253327, doi: 10.1128/AEM.67.7.3325-3327.2001.

9. El Fertas-Aissani R, Messai Y, Alouache S, Bakour R. Virulence profiles and antibiotic susceptibility patterns of importance of measures linking clinical outcomes with the enteral nutrition sector where relevant to do so. Our findings are in agreement with our aim of strengthening the interfaces between infection control and preventive actions and also between these controls and the promotion of quality within all healthcare services. The present study also endorses the need to direct healthcare funding policies towards implementation of risk control and prevention measures in strategic sectors of the healthcare services. Reflection on all the possible strategies that might contribute towards changing the current panorama of nosocomial infections, with investments in research and infection control updates, is necessary. We emphasize that data on nosocomial infections (as described in the present study and other recent studies) should be disseminated routinely to all healthcare professionals, and to hospital administration staff.
Klebsiella pneumoniae strains isolated from different clinical specimens. Pathol Biol 2013; 61: 209-216, doi: 10.1016/j. patbio.2012.10.004

10. Russo TA, Olson R, Macdonald U, Metzger D, Maltese LM, Drake EJ, et al. Aerobactin mediates virulence and accounts for increased siderophore production under iron-limiting conditions by hypervirulent (hypermucoviscous) Klebsiella pneumoniae. Infect Immun 2014; 82: 2356-2367, doi: 10.1128/IAI.01667-13.

11. Li W, Sun G, Yu Y, Li N, Chen M, Jin R, et al. Increasing occurrence of antimicrobial-resistant hypervirulent (hypermucoviscous) Klebsiella pneumoniae isolates in China. Clin Infect Dis 2014; 58: 225-232, doi: 10.1093/cid/ cit675.

12. Viale P, Giannella M, Lewis R, Trecarichi EM, Petrosillo N, Tumbarello M. Predictors of mortality in multidrug-resistant Klebsiella pneumoniae bloodstream infections. Expert Rev Anti Infect Ther 2013; 11: 1053-1063, doi: 10.1586/ 14787210.2013 .836057$.

13. Hawkey PM. Multidrug-resistant Gram-negative bacteria: a product of globalization. J Hospital Infec 2015; 89: 241-247, doi: 10.1016/j.jhin.2015.01.008.

14. Wiskur BJ, Hunt JJ, Callegan MC. Hypermucoviscosity as a virulence factor in experimental Klebsiella pneumoniae endophthalmitis. Invest Ophthalmol Vis Sci 2008; 49: 4931-4938, doi: 10.1167/iovs.08-2276.

15. Podschun R, Sievers D, Fischer A, Ullmann U. Serotypes, hemagglutinins, siderophore synthesis, and serum resistance of Klebsiella isolates causing human urinary tract infections. J Infect Dis 1993; 168: 1415-1421, doi: 10.1093/ infdis/168.6.1415.

16. Coffey A, Rombouts FM, Abee T. Influence of environmental parameters on phosphatidylcholine phospholipase $\mathrm{C}$ production in Listeria monocytogenes: a convenient method to differentiate L. monocytogenes from other Listeria species. Appl Environ Microbiol 1996; 62: 1252-1256.

17. Kokosharov T, Phetisova K. Hemolysins and aerobactin in Salmonella callinarum strains isolated from poultry. Rev Med Vet 2002; 153: 411-414. 
18. CLSI - Clinical and Laboratory Standards Institute. Performance standards for antimicrobial susceptibility testing. Approved Standard M100-S25. Clinical and Laboratory Standard Institute; 2015.

19. Vernet V, Madoulet C, Chippaux C, Philippon A. Incidence of two virulence factors (aerobactin and mucoid phenotype) among 190 clinical isolates of Klebsiella pneumoniae producing extended-spectrum beta-lactamase. FEMS Microbiol Lett 1992; 75: 1-5.

20. Yu VL, Hansen DS, Ko WC, Sagnimeni A, Klugman KP, von Gottberg A, et al. Virulence characteristics of Klebsiella and clinical manifestations of $K$. pneumoniae bloodstream infections. Emerg Infect Dis 2007; 13: 986-993, doi: 10.3201/eid1307.070187.

21. Evrard B, Balestrino D, Dosgilbert A, Bouya-Gachancard JL, Charbonnel N, Forestier C, et al. Roles of capsule and lipopolysaccharide $\mathrm{O}$ antigen in interactions of human monocyte-derived dendritic cells and Klebsiella pneumoniae. Infect Immun 2010; 78: 210-219, doi: 10.1128/IAI. 00864-09.

22. Cheng KC, Lee MF, Chuang YC, Yu WL. First description of lung abscess caused by ST23 clone capsule genotype K1 Klebsiella pneumoniae. J Formos Med Assoc 2015; 114: 379-380, doi: 10.1016/j.jfma.2013.08.008.

23. Pan YJ, Lin TL, Chen YH, Hsu CR, Hsieh PF, Wu MC, et al. Capsular types of Klebsiella pneumoniae revisited by wzc sequencing. PLoS One 2013; 8: e80670, doi: 10.1371/ journal.pone.0080670.

24. Albesa I. Klebsiella pneumoniae haemolysin adsorption to red blood cells. J Appl Bacteriol 1989; 67: 263-266.

25. Gundogan N, Citak S, Yalcin E. Virulence properties of extended spectrum beta-lactamase-producing Klebsiella species in meat samples. J Food Prot 2011; 74: 559-564, doi: 10.4315/0362-028X.JFP-10-315

26. Singh BR, Sharma VD, Chandra R. Detection, prevalence, purification and characterization of lecithinase of Klebsiella pneumoniae. Indian J Exp Biol 1999; 37: 925-932.

27. Davies J, Davies D. Origins and evolution of antibiotic resistance. Microbiol Mol Biol Rev 2010; 74: 417-433, doi: 10.1128/MMBR.00016-10.

28. Novak FR, Almeida JA, Asensi MD, Moraes BA, dos Prazeres RD. [Antimicrobial resistance of coliform isolates from expressed human milk]. Cad Saúde Pública 2001; 17: 713-717, doi: 10.1590/S0102-311X2001000300026

29. Freyne B, Carr J, Osowicki J, Steer A, Curtis N, Bryant PA. Hospital-wide rollout of antimicrobial stewardship: a stepped-wedge randomized trial. Clin Infect Dis 2015; 60: 666, doi: $10.1093 /$ cid/ciu899.

30. Eisenberg JN, Goldstick J, Cevallos W, Trueba G, Levy K, Scott J, et al. In-roads to the spread of antibiotic resistance: regional patterns of microbial transmission in northern coastal Ecuador. J R Soc Interface 2012; 9: 1029-1039, doi: 10.1098/rsif.2011.0499.

31. Salles MJC, Zurita J, Mejía C, Villegas V. Resistant Gramnegative infections in the outpatient setting in Latin America. Epidemiol Infect 2013; 141: 2459-2472, doi: 10.1017/ S095026881300191X.

32. Bonten MJ, Austin DJ, Lipsitch M. Understanding the spread of antibiotic resistant pathogens in hospitals: mathematical models as tools for control. Clin Infect Dis 2001; 33: 17391746, doi: 10.1086/323761.

33. Brasil. Ministério da Saúde. Resolução de Diretoria Colegiada (RDC) No. 20, de 5 de maio de 2011. Agência Nacional de Vigilância Sanitária - ANVISA; 2011.

34. Andremont A. Commensal flora may play key role in spreading antibiotic resistance. ASM News 2003; 69: 601-607.

35. Herrera-Leon S, Gonzalez-Sanz R, Herrera-Leon L, Echeita MA. Characterization of multidrug-resistant Enterobacteriaceae carrying plasmid-mediated quinolone resistance mechanisms in Spain. J Antimicrob Chemother 2011; 66: 287-290, doi: 10.1093/jac/dkq423.

36. Alekshun MN, Levy SB. Molecular mechanisms of antibacterial multidrug resistance. Cell 2007; 128: 1037-1050, doi: 10.1016/j.cell.2007.03.004.

37. Evans HL, Lefrak SN, Lyman J, Smith RL, Chong TW, McElearney ST, et al. Cost of Gram-negative resistance. Crit Care Med 2007; 35: 89-95, doi: 10.1097/01.CCM.000025 1496.61520.75.

38. Machado E, Coque TM, Canton R, Sousa JC, Peixe L. Antibiotic resistance integrons and extended-spectrum \{beta\}-lactamases among Enterobacteriaceae isolates recovered from chickens and swine in Portugal. J Antimicrob Chemother 2008; 62: 296-302, doi: 10.1093/jac/dkn179. 